\title{
Differential effects of aging on spatial contrast sensitivity to linear and polar sine-wave gratings
}

\author{
T.L. Costa ${ }^{1}$, R.M.T.B.L. Nogueira ${ }^{2}$, A.G.F. Pereira ${ }^{2}$ and N.A. Santos ${ }^{2}$ \\ ${ }^{1}$ Departamento de Psicologia Experimental, Instituto de Psicologia, Universidade de São Paulo, São Paulo, SP, Brasil \\ ${ }^{2}$ Departamento de Psicologia, Universidade Federal da Paraíba, João Pessoa, PB, Brasil
}

\begin{abstract}
Changes in visual function beyond high-contrast acuity are known to take place during normal aging. We determined whether sensitivity to linear sine-wave gratings and to an elementary stimulus preferentially processed in extrastriate areas could be distinctively affected by aging. We measured spatial contrast sensitivity twice for concentric polar (Bessel) and vertical linear gratings of $0.6,2.5,5$, and 20 cycles per degree (cpd) in two age groups (20-30 and 60-70 years). All participants were free of identifiable ocular disease and had normal or corrected-to-normal visual acuity. Participants were more sensitive to Cartesian than to polar gratings in all frequencies tested, and the younger adult group was more sensitive to all stimuli tested. Significant differences between sensitivities of the two groups were found for linear (only $20 \mathrm{cpd}$; $\mathrm{P}<0.01$ ) and polar gratings (all frequencies tested; $P<0.01$ ). The young adult group was significantly more sensitive to linear than to circular gratings in the $20 \mathrm{cpd}$ frequency. The older adult group was significantly more sensitive to linear than to circular gratings in all spatial frequencies, except in the $20 \mathrm{cpd}$ frequency. The results suggest that sensitivity to the two kinds of stimuli is affected differently by aging. We suggest that neural changes in the aging brain are important determinants of this difference and discuss the results according to current models of human aging.
\end{abstract}

Key words: Contrast sensitivity; Circular gratings; Linear gratings; Aging; Spatial vision

\section{Introduction}

Does normal aging specifically affect different brain areas involved in luminance information processing? Are these effects readily evaluated by psychophysical methods? The objective of the present study was to answer these questions by measuring contrast sensitivity for stimuli where the contrast modulation has different characteristics. Several kinds of stimuli have been used to estimate contrast sensitivity throughout the last decades. Here we focus on elementary stimuli that are known to activate specific spatial frequency channels separately. In this context, an elementary stimulus consists of a pattern defined by a sinusoidal modulation of luminance in space, a pattern that cannot be further decomposed into waves of other frequencies by means of a Fourier analysis. These patterns are defined in terms of frequency, amplitude, and phase. The luminance modulation pattern might differ between stimuli. Here, stimuli with luminance modulation defined by Cartesian (linear) or polar (circular concentric according to the Bessel function) coordinates were used (1-4).
Two hypotheses are central to the rationale employed in the present study and are supported by recent literature. First, sensitivity to different spatial frequency bands is distinctively altered by conditions that affect brain function, reinforcing the notion that multiple spatial frequency channels work in parallel on the codification of visual information $(5,6)$. Second, linear and circular gratings are preferentially processed in different cortical areas (7-9).

The matter of contrast sensitivity changes through normal aging has been the object of psychophysical research for decades now. In spite of the existence of studies with conflicting results (mainly in what concerns the frequencies affected and the determinants of this effect), some hypotheses find endorsement in several articles. Among these, we highlight the notion that aging affects mostly high spatial frequency processing $(10,11)$; contrast sensitivity alterations are significant after the age of $50(12-14)$, and these alterations are due not only to

Correspondence: T.L. Costa, Rua João Moura, 187/42, 05412-001 São Paulo, SP, Brasil. E-mail: e.thiagocosta@gmail.com

Received April 8, 2013. Accepted July 23, 2013. First published online October 2, 2013. 
changes in the optic components of the eye, but also to the neural components of visual processing (15-19).

The present study compares contrast sensitivity to vertical linear and circular concentric sine-wave gratings in young and older adults. The mathematical definition of these two gratings is distinct. One is defined in Cartesian coordinates with a linear unidirectional modulation in space, while the other is defined in polar coordinates according to the Bessel cylindrical function $(1,2)$, forming a concentric pattern with the maximum luminance in the center, and gradually decreasing toward the periphery. As the research of Gallant et al. $(8,20,21)$ suggest, these two stimuli also differ in the underlying cortical processing. The linear gratings are preferentially processed in the primary visual cortex (V1) while the circular gratings are preferentially processed in area V2 and V4 neurons (22).

Most research on the role of normal aging in contrast sensitivity uses linear gratings as stimuli (23). Therefore, research using non-Cartesian stimuli is needed, as there is experimental evidence in humans and primates for the significant involvement of extrastriate areas in the processing of non-Cartesian patterns $(3,7,8,24)$. If the psychophysical response to different elementary stimuli could indicate changes in different levels of the aged visual system, the employment of the methods proposed here in clinical settings could be useful in the early differential diagnosis of conditions affecting the visual cortex. Finally, with the elderly population increasing in many countries, research on the aging brain (diseased or healthy) is critical.

\section{Subjects and Methods}

\section{Subjects}

Sixteen volunteers of both genders were divided into two groups: 8 young adults from 20 to 30 (3 males, mean $\pm S D$, $23.3 \pm 2.8$ ) years of age and 8 older adults from 60 to 70 ( 2 males, $65.8 \pm 3.27$ ) years of age. All participants were free of identifiable ocular diseases and conditions such as diabetes or hypertension, having normal (Snellen 20/20) or correctedto-normal visual acuity. Before participation, the subjects signed a free and informed consent form, according to Resolução No. 196/96 of the Conselho Nacional de Saúde (Ministério da Saúde, Brazil), which determines guidelines for research involving human beings, in compliance with the Declaration of Helsinki. The Ethics Committee of UFPB approved this research.

\section{Equipment and stimuli}

All stimuli were presented on a 19-inch cathode ray tube monitor, with the screen resolution set to $1024 \times 768$ pixels and $70 \mathrm{~Hz}$ frame rate, connected to a Pentium IV computer through Bits ++ hardware (Cambridge Research Systems, England). The Bits ++ hardware increases the resolution of the monitor luminance voltage control from 8 to 14 bits, allowing a better definition of stimulus contrast. The monitor was gamma corrected using an OptiCal photometer (Cambridge Research Systems) and the mean luminance was set to 42.6 $\mathrm{cd} / \mathrm{m}^{2}$. Software developed by our laboratory in $\mathrm{C}++$ language presented the stimuli and controlled the experimental sessions.

The stimuli consisted of static achromatic gratings of $0.6,2.5,5$, and 20 cycles per degree (cpd) of visual angle and a neutral stimulus with average luminance. Circular concentric and linear vertical gratings were used (Figure 1). All stimuli were circular measuring 7.25 degrees of visual angle in diameter, calibrated for the viewing distance of $150 \mathrm{~cm}$.

\section{Procedure}

A temporal two-alternative forced-choice psychophysical task was used in a repeated measures experimental design. Thresholds were estimated by the random successive presentation of a pair of stimuli (a neutral stimulus and a test stimulus) where the participant had to indicate between them the one containing a grating. The

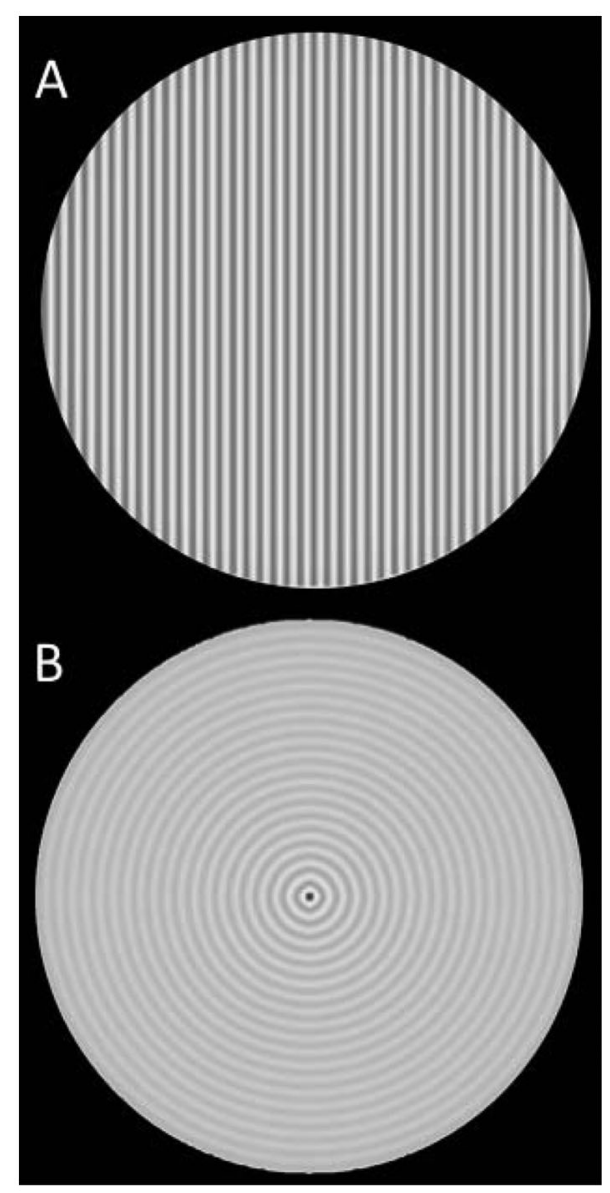

Figure 1. Examples of vertical linear $(A)$ and circular concentric $(B)$ grating stimuli used. 
estimation of the threshold for each spatial frequency involved a different experimental session for the two kinds of stimuli tested, and every experimental condition was repeated on different days. As a result, each subject was tested in four different sessions.

The experimental sessions started with a sound signal followed by the presentation of a pair of stimuli, one at a time. Each stimulus was presented for $2 \mathrm{~s}$, with a 1-s interval between them. The test stimulus was presented either first or second in a random fashion. The participants were required to press the left button of the computer mouse (marked with the number 1) when the test stimulus was presented first, and the right button (marked with the number 2) when it was presented second. A different sound signal announced when the subject response was correct. The experimental session was automatically terminated after six response reversals.

All measurements were taken binocularly and the experimental sessions started with the stimulus at suprathreshold levels, after a rehearsal trial to make sure that the task was clearly understood. The following staircase rule was applied: three consecutive correct responses led to a $20 \%$ decrease in contrast, and one wrong response led to an increase of the same percentage. This yielded a $79 \%$ probability of stimulus detection throughout the session $(24,25)$.

\section{Data analyses}

In order to study the effect of aging on response to different grating types and spatial frequencies, we analyzed the data in a 2 (age) $\times 2$ (grating type) $\times 4$ (spatial frequency) using ANOVA. We measured the effect size using partial eta squared $\left(\eta^{2}\right)$ for each interaction and, when appropriate, post hoc analyses were carried out using Bonferroni's post hoc test in the Statistica 11 software (Statsoft).

\section{Results}

Figure 2 shows the contrast sensitivity curves for the two kinds of stimuli. The young adult group had greater sensitivity for all stimuli tested. ANOVA showed a significant effect of age $\left[F_{(1,95)}=229.3, P<0.001, \eta_{p}^{2}=0.70\right]$, grating type $\left[F_{(1,95)}=244.09, P<0.001, \eta_{p}^{2}=0.71\right]$, spatial frequency $\left[\mathrm{F}_{(3,285)}=1141.5, \mathrm{P}<0.001, \eta^{2}=0.82\right]$, and $a$ significant interaction between age, grating type, and spatial frequency $\left[F_{(3,285)}=41.05, P<0.001, \eta_{p}^{2}=0.30\right]$.

The Bonferroni post hoc test showed significant differences between age groups for the vertical linear grating of $20 \mathrm{cpd}$ and for all the concentric circular gratings tested $(P<0.01)$. Post hoc analyses also indicated that young adult sensitivities for the two grating types differed for the 20-cpd frequency $(P<0.001)$, did not differ for 0.6 and $2.5 \mathrm{cpd}(P=1)$, and had a borderline significant effect at $5 \mathrm{cpd}(P=0.073)$. Nevertheless, young adults were $1.9,3.8,4.1$, and 2.2 times more
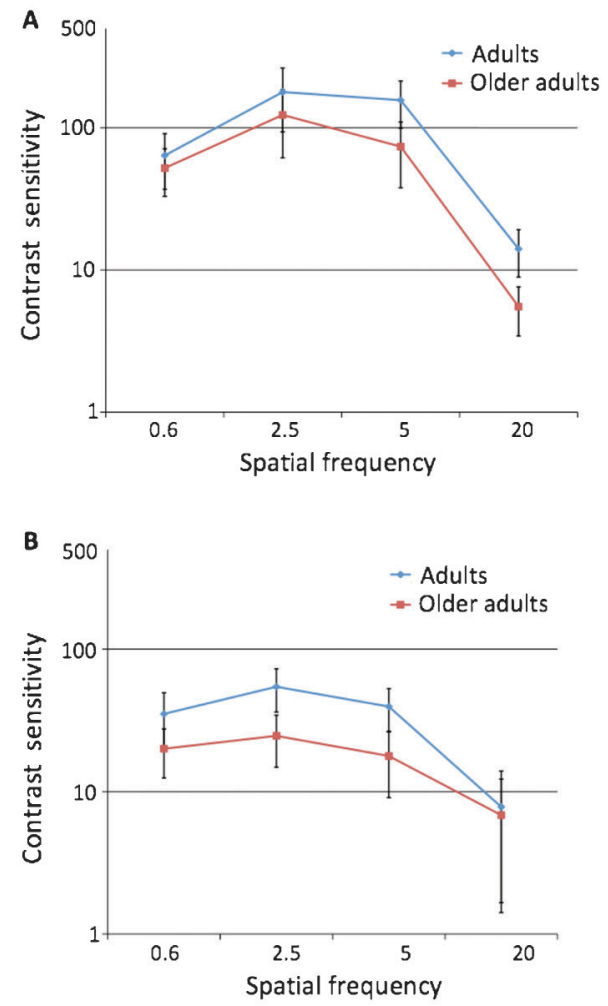

Figure 2. $A$, Contrast sensitivity curves of adults and older adults for linear gratings. $B$, Contrast sensitivity curves of the two age groups for circular concentric gratings. Data are reported as means $\pm S D$ for each frequency.

sensitive to linear than to circular gratings for the frequencies of $0.6,2.5,5$, and $20 \mathrm{cpd}$, respectively (Figure 3). The sensitivity of the older adult group for different grating types differed significantly at all frequencies $(P<0.001)$, except for $20 \mathrm{cpd}(P=0.99)$. Older adults were $2.7,5.2,4.8$, and 0.9 times more sensitive to linear than to circular gratings for the frequencies of $0.6,2.5,5$, and $20 \mathrm{cpd}$, respectively (Figure 3 ).

In summary, both young and older adults were more sensitive to linear than to circular gratings. Although sensitivity for linear and circular gratings was different in both age groups, only the older adult group showed statistically significant differences for the two grating types at the low and medium spatial frequency ranges. This is supported by the sensitivity ratio for the two grating types, where there was an increase in the ratio for the older compared with the younger adult group (Figure 3 ). The ratio of 0.9 suggests that the older adult group had practically the same sensitivity to circular and linear gratings of $20 \mathrm{cpd}$.

\section{Discussion}

Group differences for circular grating sensitivity presented here corroborate the study of Santos et al. (4), 


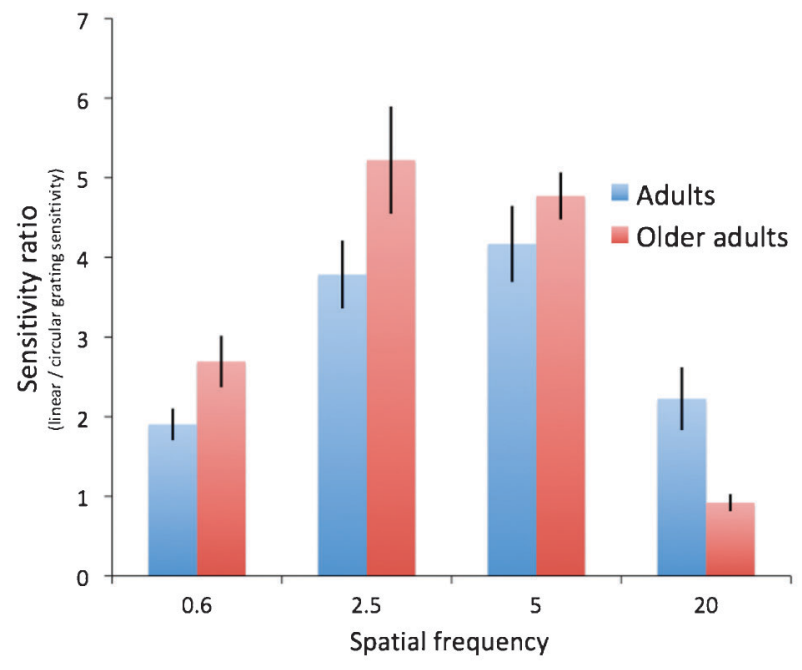

Figure 3. Sensitivity ratios of young adults (20-30 years of age) and older adults (60-70 years of age) for linear and circular gratings. Data are reported as means $\pm S D$. Values greater than one indicate that sensitivity to vertical linear was greater than for circular concentric gratings.

which reported age-related sensitivity losses to the frequencies of $0.25,1.0,2.0$, and $4.0 \mathrm{cpd}$ in mesopic luminance conditions. For the linear gratings, these authors reported the same age-related sensitivity decrease as for the circular gratings, in contrast to the data presented here. The differences between the abovementioned results might be due to the distinct luminance conditions and spatial frequency ranges employed in the two studies. The notion that, in older adults, contrast sensitivity impairment increases with decreasing background luminance and that the determinants of this phenomenon are partially neural is not new in the literature $(10,26)$. The methods employed here might be more sensitive to the parvocellular pathway function (photopic luminance, frequencies from 0.6 to $20 \mathrm{cpd}$ ), whereas those of Santos et al. (4) tend to be most indicative of the magnocellular pathway function (mesopic luminance conditions, lower spatial frequencies). Therefore, an analysis of these contrasting results and the frequency-specific effect of aging on contrast sensitivity (Figure 2) might show that human aging distinctively affects luminance contrast processing in the magno and parvocellular pathways.

To date, age-related changes in postretinal visual pathways have not been extensively evaluated, and most studies using animal models were not conclusive (27). One of the few studies using specific behavioral methods to test for losses in the magnocellular and parvocellular pathways in humans suggests that both pathways are affected significantly by normal aging and that the parvocellular is the most largely affected (15). This hypothesis is in accordance with the results presented here. It is important to note that older adults' sensitivity to $20 \mathrm{cpd}$ was practically the same for the two grating types (Figure 3). This phenomenon might not be determined by parvocellular pathway function but by changes in the normal aging eye (i.e., increased intraocular light scatter, increased optical aberrations) that could affect the processing of high spatial frequencies (for a review see Ref. 27). For more information on the magno- and parvocellular pathways see Sincich and Horton (28) and Souza et al. (6).

Older adults' loss of sensitivity to high spatial frequency Cartesian gratings was already expected, as there are several reports of that phenomenon available in the literature. Spear (23) reviews 11 articles that reported no significant changes in the sensitivity to low spatial frequencies ( $1 \mathrm{cpd}$ and below) throughout normal aging in humans. Spear concluded that there is a general consensus about the invariability of the sensitivity to the low spatial frequency band in the normal aging process. In a recent review, Owsley (27) reports that this is still true today for studies using static sine-wave gratings. It is important to note that none of these studies used non-Cartesian stimuli.

More interestingly, the data presented here suggest that normal human aging has different consequences on the sensitivity to linear and circular gratings. There are robust differences between the two kinds of gratings in that which concerns the frequency bands affected significantly by the aging process. Young adults' sensitivities for the two grating types only differed significantly for the $20 \mathrm{cpd}$, whereas older adults' sensitivities differed significantly for all frequencies, except for $20 \mathrm{cpd}$. The analysis of the sensitivity ratios for different grating types also shows differences between age groups (Figure 3) and illustrates older adults' sensitivity loss for circular concentric gratings of low and medium spatial frequencies.

These differences might have determinants at the extrastriate level. Strong evidence from neurophysiological research suggests that the Cartesian gratings are preferentially processed in V1 neurons, as the polar gratings are preferentially processed at extrastriate levels $(7,20)$. This hypothesis is in agreement not only with studies such as those by Gallant et al. $(8,20,21)$, but also with studies on the changes in sensitivity to second-order stimuli (nonelementary stimuli, defined by changes in features, texture, and depth, and not only by sinusoidal modulation of luminance in space) and studies on changes in perception of contour deformation throughout aging. The research of Habak and Faubert (18) and Tang and Zhou (29) suggest that sensitivity to second-order stimuli is significantly more affected by aging than sensitivity to first-order stimuli (defined only by luminance modulation), which is an expression of the greater complexity involved in secondorder processing. Tang and Zhou (29) also suggested that sensitivity to second-order stimuli decreases earlier than sensitivity to first-order stimuli.

The hypothesis illustrated by our results is also in accordance with the research of Legault et al. (30). It 
suggests that perception of curved shapes such as circular concentric gratings recruits a neural circuitry much more sophisticated than the one recruited by straight lines, because of the need to integrate groups of cells with distinct orientation tuning. Therefore, the aging process would tend to have a larger effect on the processing of circular patterns.

The hypotheses raised here are consistent with Faubert's (31) theory of visual perception and aging. According to Faubert, low and higher level visual functions are affected by aging, but the extent of this effect is related to the complexity of the neural circuitry involved in the task. He suggests that lower-level functions require less computational load, and a performance equivalent to a younger subject might be obtained by the recruitment of alternate neural networks. However, when this computational load reaches a certain level of complexity, larger alternate networks are required and, as a result of physiological limitations of the aging brain, performance decreases. The recruitment of alternate neural network hypothesis is consistent with the compensation-related utilization of neural circuit hypothesis suggested in the research of Reuter-Lorenz and Cappell (32) and ReuterLorenz and Lustig (33). After a review of functional brainimaging studies, these authors observed an overactivation of brain areas in older adults, leading to a performance

\section{References}

1. Kelly $\mathrm{DH}$. J-o stimulus patterns for visual research. J Opt Soc Am 1960; 50: 1115-1116, doi: 10.1364/JOSA.50. 001115.

2. Zana $\mathrm{Y}$, Cavalcanti AC. Contrast sensitivity functions to stimuli defined in Cartesian, polar and hyperbolic coordinates. Spat Vis 2005; 18: 85-98, doi: 10.1163/15685680 52801618.

3. Simas ML, Santos NA. Contrast sensitivity to radial frequencies modulated by $\mathrm{Jn}$ and jn Bessel profiles. Braz J Med Biol Res 2002; 35: 1357-1367, doi: 10.1590/S0100879X2002001100014.

4. Santos NA, Oliveira AB, Nogueira RM, Simas ML. Mesopic radial frequency contrast sensitivity function for young and older adults. Braz J Med Biol Res 2006; 39: 791-794, doi: 10.1590/S0100-879X2006000600012.

5. De Valois RL, De Valois KK. Spatial vision. New York: Oxford University Press; 1988.

6. Souza GS, Gomes BD, Saito CA, da Silva FM, Silveira LC. Spatial luminance contrast sensitivity measured with transient VEP: comparison with psychophysics and evidence of multiple mechanisms. Invest Ophthalmol Vis Sci 2007; 48: 3396-3404, doi: 10.1167/iovs.07-0018.

7. David SV, Hayden BY, Gallant JL. Spectral receptive field properties explain shape selectivity in area V4. J Neurophysiol 2006; 96: 3492-3505, doi: 10.1152/jn.00575. 2006.

8. Gallant JL, Connor CE, Rakshit S, Lewis JW, Van Essen DC. Neural responses to polar, hyperbolic, and Cartesian gratings in area V4 of the macaque monkey. J Neurophysiol equivalent to young adults in low-demand cognitive tasks. As the complexity of the task increases, the older adult brain reaches a resource ceiling, resulting in a performance decrease (32).

The results of the present study suggest that aging might have a more pronounced effect in higher-order visual areas than in $\mathrm{V} 1$ and that this phenomenon might be partially observed through psychophysical tests using circular concentric sine-wave gratings. In fact, single-unit recordings detected losses in the signal-to-noise ratio and sensitivity in cortical neurons of elderly monkeys, and these losses were even more robust in V2 than in V1 neurons $(27,34)$. Notwithstanding, it is clear that psychophysical methodology is not incontrovertible and that further research with complementary methods is needed to clarify this issue. The results presented here suggest further research on how different cortical areas involved in spatial vision might be affected not only in the aging process, but also by clinical conditions. Non-Cartesian elementary stimuli might constitute an instrument for investigations of this nature, both in clinical and basic science.

\section{Acknowledgments}

Research sponsored by CNPq, CAPES and UFPB.

9. Wang YZ. Effects of aging on shape discrimination. Optom Vis Sci 2001; 78: 447-454, doi: 10.1097/00006324-20010 6000-00019.

10. Schefrin BE, Tregear SJ, Harvey LO Jr, Werner JS. Senescent changes in scotopic contrast sensitivity. Vision Res 1999; 39: 3728-3736, doi: 10.1016/S0042-6989(99) 00072-3.

11. Fiorentini A, Porciatti V, Morrone MC, Burr DC. Visual ageing: unspecific decline of the responses to luminance and colour. Vision Res 1996; 36: 3557-3566, doi: 10.1016/ 0042-6989(96)00032-6.

12. Nio YK, Jansonius NM, Fidler V, Geraghty E, Norrby S, Kooijman AC. Age-related changes of defocus-specific contrast sensitivity in healthy subjects. Ophthalmic Physiol Opt 2000; 20: 323-334, doi: 10.1016/S0275-5408(99) 00103-9.

13. Owsley C, Sekuler R, Siemsen D. Contrast sensitivity throughout adulthood. Vision Res 1983; 23: 689-699, doi: 10.1016/0042-6989(83)90210-9.

14. Haegerstrom-Portnoy G, Schneck ME, Brabyn JA. Seeing into old age: vision function beyond acuity. Optom Vis Sci 1999; 76: 141-158, doi: 10.1097/00006324-199903000-000 14.

15. Elliott SL, Werner JS. Age-related changes in contrast gain related to the $M$ and $P$ pathways. $J$ Vis 2010; 10: 4-15, doi: 10.1167/10.4.4.

16. Elliott SL, Choi SS, Doble N, Hardy JL, Evans JW, Werner JS. Role of high-order aberrations in senescent changes in 
spatial vision. J Vis 2009; 9: 24-16, doi: 10.1167/9.2.24.

17. Pearson PM, Schmidt LA, Ly-Schroeder E, Swanson WH. Ganglion cell loss and age-related visual loss: a cortical pooling analysis. Optom Vis Sci 2006; 83: 444-454, doi: 10.1097/01.opx.0000218432.52508.10.

18. Habak C, Faubert J. Larger effect of aging on the perception of higher-order stimuli. Vision Res 2000; 40: 943-950, doi: 10.1016/S0042-6989(99)00235-7.

19. Haegerstrom-Portnoy G. The Glenn A. Fry Award Lecture 2003: Vision in elders - summary of findings of the SKI study. Optom Vis Sci 2005; 82: 87-93, doi: 10.1097/01. OPX.0000153162.05903.4C.

20. Gallant JL, Shoup RE, Mazer JA. A human extrastriate area functionally homologous to macaque V4. Neuron 2000; 27 : 227-235, doi: 10.1016/S0896-6273(00)00032-5.

21. Gallant JL, Braun J, Van Essen DC. Selectivity for polar, hyperbolic, and Cartesian gratings in macaque visual cortex. Science 1993; 259: 100-103, doi: 10.1126/science. 8418487.

22. Mahon LE, De Valois RL. Cartesian and non-Cartesian responses in LGN, V1, and V2 cells. Vis Neurosci 2001; 18: 973-981.

23. Spear PD. Neural bases of visual deficits during aging. Vision Res 1993; 33: 2589-2609, doi: 10.1016/0042-698 9(93)90218-L.

24. Santos NA, Simas MLB. Perception and visual processing of form in humans: radial frequency filters of 1 and $4 \mathrm{cpd}$. Psicol Refl Crít 2000; 15: 383-391, doi: 10.1590/S010279722002000200015.

25. Wetherill GB, Levitt $H$. Sequential estimation of points on a psychometric function. Br J Math Stat Psychol 1965; 18: 1-10, doi: 10.1111/j.2044-8317.1965.tb00689.x.

26. Sloane ME, Owsley C, Jackson CA. Aging and luminanceadaptation effects on spatial contrast sensitivity. J Opt Soc Am A 1988; 5: 2181-2190, doi: 10.1364/JOSAA.5.002181.

27. Owsley C. Aging and vision. Vision Res 2011; 51: 16101622, doi: 10.1016/j.visres.2010.10.020.

28. Sincich LC, Horton JC. The circuitry of V1 and V2: integration of color, form, and motion. Annu Rev Neurosci 2005; 28: 303-326, doi: 10.1146/annurev.neuro.28.061604. 135731.

29. Tang Y, Zhou Y. Age-related decline of contrast sensitivity for second-order stimuli: earlier onset, but slower progression, than for first-order stimuli. J Vis 2009; 9: 18, doi: 10 1167/9.7.18.

30. Legault I, Allard R, Faubert J. Normal aging and the perception of curvature shapes. Optom Vis Sci 2007; 84: 1087-1092, doi: 10.1097/OPX.0b013e31815b9e25.

31. Faubert J. Visual perception and aging. Can J Exp Psychol 2002; 56: 164-176, doi: 10.1037/h0087394.

32. Reuter-Lorenz PA, Cappell KA. Neurocognitive aging and the compensation hypotesis. Curr Direct Psychol Sci 2008; 17: 177-182, doi: 10.1111/j.1467-8721.2008.00570.x.

33. Reuter-Lorenz PA, Lustig C. Brain aging: reorganizing discoveries about the aging mind. Curr Opin Neurobiol 2005; 15: 245-251, doi: 10.1016/j.conb.2005.03.016.

34. Wang Y, Zhou Y, Ma Y, Leventhal AG. Degradation of signal timing in cortical areas $\mathrm{V} 1$ and $\mathrm{V} 2$ of senescent monkeys. Cereb Cortex 2005; 15: 403-408, doi: 10.1093/ cercor/bhh143. 